\title{
FLAT SURFACES WITH MEAN CURVATURE VECTOR OF CONSTANT LENGTH IN EUCLIDEAN SPACES
}

\author{
KAZUYUKI ENOMOTO
}

(Communicated by Jonathan M. Rosenberg)

\begin{abstract}
Complete flat surfaces in $\mathbb{R}^{n}$ are studied under the condition that the normal connection is flat and the length of the mean curvature vector is constant. It is shown that such a surface must be the product of two curves of constant geodesic curvature.
\end{abstract}

In this paper we prove the following theorem.

Theorem. Let $M$ be a complete $C^{\infty}$ flat surface in $\mathbb{R}^{n}$. Suppose that the normal connection of $M$ is flat and the length of the mean curvature vector is constant. Then there exist curves of constant geodesic curvature $C_{1}$ in $\mathbb{R}^{r}$ and $C_{2}$ in $\mathbb{R}^{n-r}$ $(1 \leq r \leq n-1)$ such that $M$ is congruent to the Riemannian product of $C_{1}$ and $C_{2}$.

A surface is called flat if the Gaussian curvature is zero at every point.

Let $M$ be a surface in $\mathbb{R}^{n}$. We denote the standard inner product and the covariant differentiation of $\mathbb{R}^{n}$ by $\langle$,$\rangle and \bar{D}$ respectively. For tangent vector fields $X, Y$, and a normal vector field $\xi$ of $M$, we write $\bar{D}_{X} Y=$ $D_{X} Y+B(X, Y)$ and $\bar{D}_{X} \xi=-A_{\xi} X+D_{X}^{\perp} \xi$, where $D_{X} Y$ (resp. $-A_{\xi} X$ ) is the tangential component of $\bar{D}_{X} Y$ (resp. $\left.\bar{D}_{X} \xi\right)$, and $B(X, Y)$ (resp. $\left.D_{X}^{\perp} \xi\right)$ is the normal component of $\bar{D}_{X} Y$ (resp. $\left.\bar{D}_{X} \xi\right)$. Let $\left\{e_{i}\right\} \quad(i=1,2)$ be a local orthonormal frame field of the tangent bundle $T M$ of $M$ and $\left\{e_{\alpha}\right\} \quad(\alpha=$ $3, \ldots, n)$ be a local orthonormal frame field of the normal bundle $T^{\perp} M$ of $M$. We define $\omega_{A B}(X)=\left\langle\bar{D}_{X} e_{A}, e_{B}\right\rangle(A, B=1, \ldots, n)$ for $X$ in $T M$. We say that a point $p$ in $M$ is umbilical with respect to a normal vector $\xi$ at $p$ if $A_{\xi}$ is proportional to the identity transformation of the tangent space $T_{p} M$ of $M$ at $p$. If $K$ denotes the Gaussian curvature of $M$, the Gauss equation is given by

$$
K=\left\langle B\left(e_{1}, e_{1}\right), B\left(e_{2}, e_{2}\right)\right\rangle-\left\langle B\left(e_{1}, e_{2}\right), B\left(e_{1}, e_{2}\right)\right\rangle .
$$

Received by the editors July 28, 1989 and, in revised form, October 19, 1989.

1980 Mathematics Subject Classification (1985 Revision). Primary 53C42; Secondary 53A07.

Key words and phrases. Flat surface, mean curvature vector, product surface. 
The Codazzi equation is given by

$$
\left(D_{X} B\right)(Y, Z)-\left(D_{Y} B\right)(X, Z)=0
$$

for tangent vector fields $X, Y$, and $Z$.

Lemma 1. If $M$ is flat and the normal connection of $M$ is flat, then there exists an orthonormal basis $\left\{e_{1}, e_{2}\right\}$ of $T_{p} M$ for each $p$ in $M$ such that $B\left(e_{1}, e_{2}\right)=$ $B\left(e_{2}, e_{1}\right)=0$ and $\left\langle B\left(e_{1}, e_{1}\right), B\left(e_{2}, e_{2}\right)\right\rangle=0$.

Proof. Since the normal connection is fiat, all $A_{\xi}$ 's $\left(\xi \in T_{p}^{\perp} M\right)$ are simultaneously diagonalizable. Hence there exists an orthonormal basis $\left\{e_{1}, e_{2}\right\}$ of $T_{p} M$ such that $\left\langle A_{\xi} e_{1}, e_{2}\right\rangle=\left\langle A_{\xi} e_{2}, e_{1}\right\rangle=0$ for all $\xi$. Then we have $B\left(e_{1}, e_{2}\right)=B\left(e_{2}, e_{1}\right)=0$. Since the Gaussian curvature is zero, it follows from (1) and $B\left(e_{1}, e_{2}\right)=0$ that $\left\langle B\left(e_{1}, e_{1}\right), B\left(e_{2}, e_{2}\right)\right\rangle=0$.

Let $M$ be a surface in $\mathbb{R}^{n}$ which satisfies the conditions in the theorem. Since the length of the mean curvature vector is constant, we have $\left\|B\left(e_{1}, e_{1}\right)\right\|^{2}+$ $\left\|B\left(e_{2}, e_{2}\right)\right\|^{2}=c^{2}$ for some constant $c$, where $\left\{e_{1}, e_{2}\right\}$ is the orthonormal basis of $T_{p} M$ given in Lemma 1 .

If $c=0$, then $M$ is totally geodesic. In the following argument, we assume $c \neq 0$.

Let $\pi: \widetilde{M} \rightarrow M$ be the universal covering of $M$. Since $M$ is complete and flat, $\widetilde{M}$ is isometric to $\mathbb{R}^{2}$ equipped with the standard flat metric.

Lemma 2. There exists a global $C^{\infty}$ orthonormal frame field $\left\{\tilde{e}_{1}, \tilde{e}_{2}\right\}$ on $\widetilde{M}$ such that, for any $\tilde{p}$ in $\widetilde{M}, d \pi\left(\tilde{e}_{1}\right)$ and $d \pi\left(\tilde{e}_{2}\right)$ are eigenvectors of $A_{\xi}$ for all $\xi$ in the normal space of $M$ at $\pi(\tilde{p})$.

Proof. Since $c \neq 0$, there does not exist a point on $M$ which is umbilical with respect to all normal vectors. Hence for each point $p$ of $M$ there exists a simply connected neighborhood $U$ and a $C^{\infty}$ normal vector field $\xi$ defined on $U$ such that every point in $U$ is not umbilical with respect to $\xi$. Let $\left\{e_{1}, e_{2}\right\}$ be an orthonormal frame field of $\left.T M\right|_{U}$ which consists of eigenvectors of $A_{\xi}$. By a result in [6], $\left\{e_{1}, e_{2}\right\}$ is a $C^{\infty}$ frame field on $U$. For $q$ in $M$, let $V$, $\eta,\left\{f_{1}, f_{2}\right\}$ have the same properties as $U, \xi,\left\{e_{1}, e_{2}\right\}$ respectively. Suppose that $U \cap V$ is nonempty and connected. Since the normal connection of $M$ is flat, $A_{\xi}$ and $A_{\eta}$ have common eigenvectors on $U \cap V$. Hence we can take $\left\{f_{1}, f_{2}\right\}$ so that $e_{1}=f_{1}$ and $e_{2}=f_{2}$ on $U \cap V$. Since $\widetilde{M}$ is simply connected, this continuation method and the standard monodromy argument allows us to define a global $C^{\infty}$ orthonormal frame field $\left\{\tilde{e}_{1}, \tilde{e}_{2}\right\}$ on $\widetilde{M}$ which has the desired property.

By Lemma 2, $B\left(d \pi\left(\tilde{e}_{i}\right), d \pi\left(\tilde{e}_{i}\right)\right) \quad(i=1,2)$ is a $C^{\infty}$ normal vector field of $M$ which satisfies $\left\|B\left(d \pi\left(\hat{e}_{1}\right), d \pi\left(\tilde{e}_{1}\right)\right)\right\|^{2}+\left\|B\left(d \pi\left(\tilde{e}_{2}\right), d \pi\left(\tilde{e}_{2}\right)\right)\right\|^{2}=c^{2}$. We use the Codazzi equation (2) to obtain

$$
D_{e_{2}}^{\perp}\left(B\left(e_{1}, e_{1}\right)\right)=\omega_{12}\left(e_{1}\right)\left(B\left(e_{1}, e_{1}\right)-B\left(e_{2}, e_{2}\right)\right)
$$


and

$$
D_{e_{1}}^{\perp}\left(B\left(e_{2}, e_{2}\right)\right)=\omega_{21}\left(e_{2}\right)\left(B\left(e_{2}, e_{2}\right)-B\left(e_{1}, e_{1}\right)\right),
$$

where $e_{i}=d \pi\left(\tilde{e}_{i}\right)$ for $i=1,2$. We define subsets $\widetilde{M}_{0}, \widetilde{M}_{1}, \widetilde{M}_{2}$ of $\widetilde{M}$ by $\widetilde{M}_{i}=\left\{\tilde{p} \in \widetilde{M}: B\left(d \pi\left(\tilde{e}_{i}\right), d \pi\left(\tilde{e}_{i}\right)\right)=0\right\}$ for $i=1,2$ and $\widetilde{M}_{0}=\widetilde{M} \backslash\left(\widetilde{M}_{1} \cup \widetilde{M}_{2}\right)$. For $\tilde{p}$ in $\widetilde{M}$ let $\tilde{\sigma}_{i}(t)$ be the maximal integral curve of $\tilde{e}_{i}$ with $\tilde{\sigma}_{i}(0)=\tilde{p}_{i}$.

Lemma 3. If $\tilde{p}$ lies in $\widetilde{M}_{1}$ (resp. $\widetilde{M}_{2}$ ), then $\tilde{\sigma}_{2}$ (resp. $\left.\tilde{\sigma}_{1}\right)$ is entirely contained in $\widetilde{M}_{1}$ (resp. $\widetilde{M}_{2}$ ). Moreover, $\tilde{\sigma}_{2}$ (resp. $\left.\tilde{\sigma}_{1}\right)$ is a geodesic of $\widetilde{M}$.

Proof. Suppose $\tilde{p}$ lies in $\widetilde{M}_{1}$. Set

$$
f(t)=\left\|B\left(d \pi\left(\tilde{e}_{1}\left(\tilde{\sigma}_{2}(t)\right)\right), d \pi\left(\tilde{e}_{1}\left(\tilde{\sigma}_{2}(t)\right)\right)\right)\right\|^{2} .
$$

Using (3), we see that $f(t)$ satisfies a differential equation

$$
d f / d t=\psi(t) f(t)
$$

where $\psi(t)=2\left\langle\bar{D}_{\tilde{e}_{1}} \tilde{e}_{1}, \tilde{e}_{2}\right\rangle\left(\tilde{\sigma}_{2}(t)\right)$. Since, by Lemma $2, \psi$ is a $C^{\infty}$ function defined for all $t$ and $f(0)=0,(5)$ implies that $f(t)=0$ for all $t$. Thus $\tilde{\sigma}_{2}(t)$ is contained in $\widetilde{M}_{1}$ for all $t$.

Since $\left\|B\left(d \pi\left(\tilde{e}_{2}\right), d \pi\left(\tilde{e}_{2}\right)\right)\right\|^{2}$ attains its maximum $\left(=c^{2}\right)$ along $\tilde{\sigma}_{2}(t)$, we have

$$
\tilde{e}_{1}\left(\left\|B\left(d \pi\left(\tilde{e}_{2}\right), d \pi\left(\tilde{e}_{2}\right)\right)\right\|^{2}\right)=0
$$

at every point of $\tilde{\sigma}_{2}(t)$. Equations (4) and (6) yield $\omega_{21}\left(e_{2}\right)=0$, which implies that $\tilde{\sigma}_{2}(t)$ is a geodesic of $\widetilde{M}$. The proof is similar when $\tilde{p}$ lies in $\widetilde{M}_{2}$.

We define continuous unit vector fields $\widetilde{Y}_{1}$ and $\widetilde{Y}_{2}$ on $\widetilde{M}$ by

$$
\tilde{Y}_{1}=\left(\| B\left(d \pi\left(\tilde{e}_{1}\right), d \pi\left(\tilde{e}_{1}\right) \| / c\right) \tilde{e}_{1}-\left(\left\|B\left(d \pi\left(\tilde{e}_{2}\right), d \pi\left(\tilde{e}_{2}\right)\right)\right\| / c\right) \tilde{e}_{2}\right.
$$

and

$$
\tilde{Y}_{2}=\left(\left\|B\left(d \pi\left(\tilde{e}_{1}\right), d \pi\left(\tilde{e}_{1}\right)\right)\right\| / c\right) \tilde{e}_{1}+\left(\left\|B\left(d \pi\left(\tilde{e}_{2}\right), d \pi\left(\tilde{e}_{2}\right)\right)\right\| / c\right) \tilde{e}_{2} .
$$

$\tilde{Y}_{1}$ and $\tilde{Y}_{2}$ are $C^{\infty}$ on $\widetilde{M}_{0}$. Moreover, using (3) and (4) together with $\left\|B\left(d \pi\left(\tilde{e}_{1}\right), d \pi\left(\tilde{e}_{1}\right)\right)\right\|^{2}+\left\|B\left(d \pi\left(\tilde{e}_{2}\right), d \pi\left(\tilde{e}_{2}\right)\right)\right\|^{2}=c^{2}$, one can show that $D_{\widetilde{Y}_{i}} \tilde{Y}_{i}=$ 0 for $i=1,2$. Thus we have

Lemma 4. Every integral curve of $\widetilde{Y}_{i} \mid \widetilde{M}_{0}(i=1,2)$ is geudesic.

Let $\tilde{p}$ be a point in $\widetilde{M}_{0}$ and let $\tilde{\gamma}_{i}(t)$ be an integral curve of $\widetilde{Y}_{i} \mid \widetilde{M}_{0}$ with $\tilde{\gamma}_{i}(0)=\tilde{p}$.

Lemma 5. $\tilde{\gamma}_{i}$ is defined for all $t \in \mathbb{R}$.

Proof. Suppose $\tilde{\gamma}_{i}$ is defined for all $t \in[0, T)$ but not for $t=T$. Then $\tilde{q}=\lim _{t \rightarrow T} \tilde{\gamma}_{i}(t)$ is contained in either $\widetilde{M}_{1}$ or $\widetilde{M}_{2}$. Suppose $\tilde{q}$ lies in $\widetilde{M}_{1}$. By 
Lemma $4, \tilde{\gamma}_{i}([0, T))$ is a geodesic segment on $\widetilde{M}$ such that $\lim _{t \rightarrow T}\left(d \tilde{\gamma}_{i} / d t\right)=$ $\lim _{t \rightarrow T} \tilde{Y}_{i}\left(\tilde{\gamma}_{i}(t)\right)= \pm \tilde{e}_{2}(\tilde{q})$. By Lemma 3 , the geodesic of $\widetilde{M}^{t \rightarrow T}$ which passes through $\tilde{q} \in \widetilde{M}_{1}$ and has the tangent vector $\pm \tilde{e}_{2}$ at $\tilde{q}$ is entirely contained in $\widetilde{M}_{1}$. This implies that $\tilde{\gamma}_{i}([0, T))$ is contained in $\widetilde{M}_{1}$, which is contradiction.

Proof of theorem. If $c=0, M$ is totally geodesic, i.e., a two-dimensional affine subspace in $\mathbb{R}^{n}$.

Suppose $c \neq 0$ and $\widetilde{M}_{0} \neq \phi$. By Lemmas 4 and 5 , every integral curve of $\widetilde{Y}_{i} \mid \widetilde{M}_{0} \quad(i=1,2)$ is a complete geodesic. Since $\widetilde{M}$ is isometric to the Euclidean plane, all integral curves of $\widetilde{Y}_{i} \mid \widetilde{M}_{0}$ for each $i$ must be parallel lines. This implies that $\widetilde{M}_{0}=\widetilde{M}$ and that the angle between $\widetilde{Y}_{1}$ and $\widetilde{Y}_{2}$ is constant on $\widetilde{M}$. Then, by the definition of $\widetilde{Y}_{i}$, we see that all integral curves of $\tilde{e}_{i}$ are parallel lines on $\widetilde{M}$ for each $i$. In the case when $\widetilde{M}_{0}=\phi$ (i.e., $\widetilde{M}=\widetilde{M}_{1}$ or $\left.\widetilde{M}=\widetilde{M}_{2}\right)$, we use Lemma 3 to observe that all integral curves of $\tilde{e}_{i}$ are parallel lines on $\widetilde{M}$. Therefore, there is a Cartesian coordinate system $\left(\tilde{u}_{1}, \tilde{u}_{2}\right)$ on $\widetilde{M}$ $\left(=\mathbb{R}^{2}\right)$ such that $\partial / \partial \tilde{u}_{i}=\tilde{e}_{i}$ for $i=1,2$. Let $\tilde{X}\left(\tilde{u}_{1}, \tilde{u}_{2}\right)$ be the position vector of the point on $\frac{\tilde{M}}{}$ whose coordinate is $\left(\tilde{u}_{1}, \tilde{u}_{2}\right)$. Then we have

$$
\partial^{2} \tilde{X} / \partial \tilde{u}_{1} \partial \tilde{u}_{2}=\bar{D}_{\tilde{e}_{1}} \tilde{e}_{2}=\bar{D}_{e_{1}} e_{2}=D_{e_{1}} e_{2}+B\left(e_{1}, e_{2}\right)=0 .
$$

Hence there exist $\mathbb{R}^{n}$-valued functions $\widetilde{C}\left(\tilde{u}_{1}\right)$ and $\widetilde{C}_{2}\left(\tilde{u}_{2}\right)$ such that $\widetilde{X}\left(\tilde{u}_{1}, \tilde{u}_{2}\right)$ $=\tilde{C}_{1}\left(\tilde{u}_{1}\right)+\tilde{C}_{2}\left(\tilde{u}_{2}\right)$. Since $\tilde{e}_{i}=\partial \tilde{X} / \partial \tilde{u}_{i}=d \widetilde{C}_{i} / d \tilde{u}_{i}$ and $\left\langle\tilde{e}_{1}, \tilde{e}_{2}\right\rangle=0$, we have

$$
\left\langle d \widetilde{C}_{1} / d \tilde{u}_{1}, d \tilde{C}_{2} / d \tilde{u}_{2}\right\rangle=0
$$

for any $\left(\tilde{u}_{1}, \tilde{u}_{2}\right)$ in $\mathbb{R}^{2}$.

Let $P_{i}$ be the affine subspace of the lowest dimension which contains $\tilde{C}_{i}$. Equation (7) implies that $P_{1}$ and $P_{2}$ are orthogonal. Since $\widetilde{X}\left(\tilde{u}_{1}, \tilde{u}_{2}\right)=$ $\widetilde{C}_{1}\left(\tilde{u}_{1}\right)+\widetilde{C}_{2}\left(\tilde{u}_{2}\right), \widetilde{M}$ is the Riemannian product of $\widetilde{C}_{1}$ and $\widetilde{C}_{2}$. Since $\widetilde{M}$ is complete, $\widetilde{C}_{i}$ is either a curve of infinite length without endpoints or a covering of a closed curve $C_{i}$. Then $M$ is the Riemannian product of $C_{1}$ and $C_{2}$. (We set $C_{i}=\widetilde{C}_{i}$ if $\widetilde{C}_{i}$ is not a covering of a closed curve.) The geodesic curvature of $\tilde{C}_{i}$ is given by $\left\|B\left(d \pi\left(\tilde{e}_{i}\right), d \pi\left(\tilde{e}_{i}\right)\right)\right\|$, which is constant since the angle between $\widetilde{Y}_{1}$ and $\widetilde{Y}_{2}$ is constant on $\widetilde{M}$. Hence $C_{1}$ and $C_{2}$ have constant geodesic curvatures.

Corollary. Let $M$ be a compact flat surface in $\mathbb{R}^{4}$. If the normal connection of $M$ is flat and the length of the mean curvature vector is constant, then $M$ is congruent to the Riemannian product of two circles, $S^{1}\left(r_{1}\right) \times S^{1}\left(r_{2}\right)$.

Remark 1. Our theorem is global (i.e., completeness is necessary), as we see in the following example: Let $S=\{(x, y, z)=(r \cos \theta, r \sin \theta, r): 1<r<2$, $0 \leq \theta<2 \pi\}$. $S$ is a flat surface in $\mathbb{R}^{3}$ whose mean curvature is $1 / 2 \sqrt{2} r$. Let $\phi(s)=\left(\phi_{1}(s), \phi_{2}(s)\right)$ be a curve in $\mathbb{R}^{2}$ parameterized by arc-length $s$ whose 
curvature is $\sqrt{2\left(1-s^{-2}\right)}$. Define an isometric immersion $\Phi: \mathbb{R}^{3} \rightarrow \mathbb{R}^{4}$ by $\Phi(x, y, z)=\left(x, y, \phi_{1}(z), \phi_{2}(z)\right)$. Then $M=\Phi(S)$ is a flat surface in $\mathbb{R}^{4}$ having a mean curvature vector of constant length and flat normal connection. But clearly, $M$ is not a Riemannian product of two curves.

Remark 2. If the mean curvature vector is parallel with respect to the normal connection, the length of the mean curvature vector is constant. Moreover, the normal connection is flat if $M$ is in $\mathbb{R}^{4}$ and not minimal. A flat surface in $\mathbb{R}^{4}$ with parallel mean curvature vector is locally the Riemannian product of two circles [4].

Remark 3. There are many compact flat surfaces in $\mathbb{R}^{4}$ with flat normal connection which are not congruent to the Riemannian product of two plane curves $[3,5]$.

Remark 4. A similar problem is studied in [1] for surfaces of positive constant curvature.

\section{REFERENCES}

1. K. Enomoto, Umbilical points on surfaces in $\mathbb{R}^{N}$, Nagoya Math. J. 100 (1985), 135-143.

2. __ The Gauss image of flat surfaces in $\mathbb{R}^{4}$, Kodai Math. J. 9 (1986), 19-32.

3. $\ldots$, Global properties of the Gauss image of flat surfaces in $\mathbb{R}^{4}$, Kodai Math. J. 10 (1987), 272-284.

4. D. A. Hoffmann, Surfaces of constant mean curvature in manifolds of constant curvature, J. Differential Geom. 8 (1973), 161-176.

5. U. Pinkall, Hopf tori in $S^{3}$, Invent. Math. 81 (1985), 379-386.

6. H. Reckziegel, Completeness of curvature surfaces of an isometric immersion, J. Differential Geom. 14 (1979), 7-20.

Faculty of Industrial Science and Technology, Science University of Tokyo, OsHAMANBE, HoKKaIDO, 049-35 JAPAN 